Nouvelles perspectives en sciences sociales

Accessibilité et offre active. Santé et services sociaux en

contexte linguistique minoritaire, Marie Drolet, Pier Bouchard

et Jacinthe Savard (dir.), Ottawa, Les Presse de l'Université

d'Ottawa, coll. Santé et société ", 2017, 414 p.

Roger Gervais

Volume 14, numéro 1, novembre 2018

URI : https://id.erudit.org/iderudit/1056438ar

DOI : https://doi.org/10.7202/1056438ar

Aller au sommaire du numéro

Éditeur(s)

Prise de parole

ISSN

1712-8307 (imprimé)

1918-7475 (numérique)

Découvrir la revue

Citer ce compte rendu

Gervais, R. (2018). Compte rendu de [Accessibilité et offre active. Santé et services sociaux en contexte linguistique minoritaire, Marie Drolet, Pier Bouchard et Jacinthe Savard (dir.), Ottawa, Les Presse de l'Université d'Ottawa, coll. Santé et société ", 2017, 414 p.] Nouvelles perspectives en sciences sociales, 14(1), 269-273. https://doi.org/10.7202/1056438ar 


\section{Comptes-rendus de lecture}

\section{Accessibilité et offre active. Santé et services sociaux en contexte linguistique minoritaire}

Marie Drolet, Pier Bouchard et Jacinthe Savard (dir.), Ottawa, Les Presse de l'Université d'Ottawa, coll. Santé et société », 2017, $414 \mathrm{p}$.

\section{Par Roger Gervais}

Université Sainte-Anne, Pointe-de-l'Église (Nouvelle-Écosse)

C elon sa Constitution, le Canada a deux langues officielles, le $\mathcal{O}$ français et l'anglais. Alors que cette loi donne l'impression que les francophones et les anglophones dans ce pays sont traités de manière équitable, de nombreux articles et livres soulignent la non-parité entre ces deux groupes : les anglophones, étant majoritaires, vivent dans une société à leur image et ont accès à plus de services; les francophones, étant minoritaires, doivent revendiquer des droits tout en combattant l'assimilation linguistique et culturelle. Pendant longtemps, ces revendications se faisaient dans l'espoir d'instituer (ou de préserver) des systèmes scolaires et postsecondaires en français. De plus, on encourageait fortement les francophones en situation minoritaire à demander des services en français afin de signaler un besoin et, par ricochet, d'inciter les institutions publiques et privées à offrir plus de 
services dans la langue de Molière. De nombreux changements se sont produits, toutefois, pour modifier la structure de ces revendications : par exemple, les revendications pour l'éducation en français se sont étendues aux services en santé en français et au lieu d'encourager les francophones à réclamer des services dans leur langue, on a commencé à encourager les institutions et les professionnels de la santé à offrir les services dans les deux langues officielles, ce qu'on appellera l'offre active. C'est dans le but de rapporter, de documenter et donc de transmettre la situation de l'offre active des services sociaux et de santé en français aux communautés francophones en situation minoritaire (CFSM), que Marie Drolet, Pier Bouchard et Jacinthe Savard ont dirigé un collectif multidisciplinaire intitulé : Accessibilité et offre active. Santé et services sociaux en contexte linguistique minoritaire.

Vingt-huit auteurs signent les nombreux chapitres qui composent ce collectif. En plus d'une introduction et d'une section dédiée à la problématique générale, ce livre se divise en cinq parties et une conclusion. On y traite de l'engagement des acteurs, des leviers politiques et juridiques, des enjeux et des besoins liés à l'accessibilité et à l'offre active des services en français. On y aborde la question de l'expérience des professionnels bilingues et on y signale les enjeux et les stratégies de formation de ces derniers. Certaines sections sont plus théoriques, alors que d'autres se réfèrent à l'empirie pour y décrire l'état de la situation. Et presque partout dans cet ouvrage collectif, on trouve une pensée centrée sur l'acteur, sur l'action et sur la stratégie. La postface affirme que c'est en se dirigeant vers l'action que l'on peut " contribuer à la formation de leaders capables d'intervenir efficacement".

Il y a tellement de perles dans ce livre. Il est difficile de ne s'arrêter qu'à quelques-unes. Par exemple, les personnes qui ne sont pas familières avec la santé des populations en situation minoritaire seront surprises d'apprendre que les personnes qui appartiennent à ces ensembles prennent plus de temps à guérir que celles qui font partie du groupe majoritaire. Selon le chapitre de la Problématique générale..., une moins bonne compréhension 
de la langue majoritaire affecte négativement le diagnostic, la réussite des plans de traitement et, par ricochet, le temps de guérison.

En lisant le chapitre intitulé Services de santé en français au Canada, on apprend que, dans une situation minoritaire, la Constitution du Canada ne garantit pas une offre homogène des services de santé en français dans le pays; « [c]e sont les provinces et les territoires qui ont principalement la responsabilité de la prestation de soins de santé et de leur dimension linguistique " (p. 94). Par conséquent, dans tout le pays, seul le NouveauBrunswick offre un droit général à des soins de santé dans sa langue, alors que d'autres provinces n'offrent qu'un bureau des affaires francophones qui conseille, informe et traduit selon les besoins.

Dans le chapitre 8, plus de 72 intervenants bilingues en santé rapportent de nombreuses difficultés associées à l'offre de service de santé en français et en anglais au Canada. Parmi ces difficultés, on trouve une pénurie de professionnels et de services bilingues; les défis de repérage de la langue préférée par le client; les défis de repérage de collègues, de professionnels et de services bilingues; et le manque d'appui pour offrir des services dans la langue officielle de la minorité.

Chaque chapitre présente des informations fort utiles, même essentielles, pour les personnes intéressées par le fait minoritaire et par les services de santé offerts aux minorités.

Si nous avons un reproche à faire à ce livre, c'est son dévouement au modèle centré sur un humain qui est toujours autonome, rationnel, stratégique et calculateur, ce qu'on appelle en sociologie un modèle centré sur l'acteur. Il est vrai qu'une intervention sociétale efficace exige un plan stratégique. Il est vrai également qu'un argumentaire doit se solidifier avec des données probantes, ce que ce livre offre en grande quantité. La raison et la stratégie fournissent des moyens efficaces pour répondre aux gestionnaires qui, entre autres : «[...] résistent au changement et contestent la nécessité de planifier des services en français sous prétexte que la demande est trop faible » (p. 57). Toutefois, l'étude de la santé 
et de l'offre active se limite beaucoup si elle reste dans une perspective anthropocentrique. Les nombreux arguments contre le tabagisme n'ont jamais suffi pour arrêter tous les fumeurs. Dans la même veine, connaître les risques associés à une mauvaise habitude alimentaire et à une vie inactive réduit peu les problèmes d'embonpoint des sociétés postmodernes. Ou encore, les nombreuses campagnes de sensibilisation n'arrivent toujours pas à éliminer les maladies transmises sexuellement ou la maternité précoce. En gros, les modèles qui traitent l'humain comme une entité simplement raisonnable et stratégique ont toujours eu du mal à bien cerner les comportements humains, et donc à susciter le changement. La persistance de l'anthropocentrisme nous étonne surtout puisque la psychologie sociale et la sociologie relationnelle signalent, depuis longtemps, ses lacunes - voir, par exemple, le modèle tripartite de Rosenberg révisé par Zanna et Rempel en $1988^{1}$ ou alors les nombreux écrits sur l'émoraison de Simon Laflamme ${ }^{2}$.

Il est avantageux et possible de concevoir un humain qui est émotion et raison, individuel et social, historique et synchronique. Cette conception que nous proposons est d'ailleurs repérable dans ce collectif. En effet, à lire les pages du collectif Accessibilité et offre active, on trouve de nombreux passages qui révèlent la riche complexité de l'humain. Par exemple, dans la citation qui suit, on voit l'humain qui justifie ses décisions en relation avec autrui et en relation avec ses émotions :

Plusieurs personnes rencontrées [lors des entrevues] ont relevé, par divers exemples, la situation de l'usager qui n'ose demander le service en français par crainte d'être considéré comme un fauteur de troubles et que cela nuise éventuellement au traitement de son dossier. En effet, il est important de rappeler que, dans bien des cas, notamment lors d'un séjour en milieu hospitalier, l'usager se sent vulnérable, stressé et dépendant des professionnels. Parfois intimidé par le statut de ceux-ci,

$1 \quad$ Mark P. Zanna, M.P. et John Karl Rempel, « Attitudes: A New Look at an Old Concept ", dans Daniel Bar-Tal et Arie W. Kruglanski (dir.), The Social Psychology of Knowledge, Cambridge, Cambridge University Press, 1988, p. 315-334.

2 Par exemple, Communication et émotion. Essai de microsociologie relationnelle, Paris, L'Harmattan, coll. "Logiques sociales », 1995. 
il n'osera pas toujours les questionner et encore moins exiger qu'on lui fournisse des explications dans sa langue (p. 57).

De plus, les auteurs de l'introduction citent des nombreux travaux indiquant la "valeur affective plus large, complexe et spontanée " liée aux mots de la première langue apprise ( $\mathrm{p}$. 18). Pourquoi alors se limiter tout simplement à l'étude de l'acteur rationnel? Les modèles qui saisissent la complexité humaine et ces éléments affectifs existent. Pourquoi ne pas les exploiter? Il y a beaucoup à gagner en étudiant l'humain relationnel.

Accessibilité et offre active. Santé et services sociaux en contexte linguistique minoritaire révèle l'importance de la composante linguistique en santé. En plus d'être nécessaire pour le bien-être d'une population, offrir des services dans la langue d'un groupe minoritaire assure un système juste et équitable. Les conséquences de ne pas offrir ces services sont la réduction de la confiance dans le système de santé, un temps de guérison inégal entre les différents groupes sociétaux, des services plus dispendieux et des chances accrues d'épuisement chez les professionnels bilingues. Malgré l'application d'un modèle centré sur l'acteur, ce livre est un atout pour les personnes qui œuvrent dans ou qui étudient le domaine de la santé. 